\title{
edoc
}

Institutional Repository of the University of Basel

University Library

Schoenbeinstrasse 18-20

CH-4056 Basel, Switzerland

http://edoc.unibas.ch/

\section{Coherent optical transitions in implanted nitrogen vacancy centers}

Chu, Y. and de Leon, N. P. and Shields, B. J. and Hausmann, B. and Evans, R. and Togan, E. and Burek, M. J. and Markham, M. and Stacey, A. and Zibrov, A. S. and Yacoby, A. and Twitchen, D. J. and Loncar, M. and Park, H. and Maletinsky, P. and Lukin, M. D.

Posted at edoc, University of Basel

Official URL: http://edoc.unibas.ch/dok/A6337981

Originally published as:

Chu, Y. and de Leon, N. P. and Shields, B. J. and Hausmann, B. and Evans, R. and Togan, E. and Burek, M. J. and Markham, M. and Stacey, A. and Zibrov, A. S. and Yacoby, A. and Twitchen, D. J. and Loncar, M. and Park, H. and Maletinsky, P. and Lukin, M. D.. (2014) Coherent optical transitions in implanted nitrogen vacancy centers. Nano letters, Vol. 14, H. 4. S. 1982-1986.

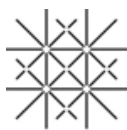




\title{
Coherent optical transitions in implanted nitrogen vacancy centers
}

\author{
Y. Chu ${ }^{*, \dagger}, \#$ N.P. de Leon, ${ }^{\dagger}, \#$ B.J. Shields, ${ }^{\dagger}$ B. Hausmann, ${ }^{\ddagger}$ R. Evans, ${ }^{\dagger}$ E. \\ Togan, ฯ M. J. Burek, ${ }^{\ddagger}$ M. Markham,$^{\S}$ A. Stacey, ${ }^{\S}$ A.S. Zibrov ${ }^{\dagger}$ A. Yacoby, ${ }^{\dagger}$ \\ D.J. Twitchen, ${ }^{\S}$ M. Loncar, ${ }^{\dagger}$ H. Park, ${ }^{\dagger}$ P. Maletinsky, ${ }^{\perp}$ and M.D. Lukin ${ }^{\dagger}$ \\ Department of Physics, Harvard University, Cambridge, MA 02138, School of Engineering \\ and Applied Science, Harvard University, Cambridge, MA 02138, Institute for Quantum \\ Electronics, ETH-Zurich, CH-8093, Switzerland, Element Six Ltd, Kings Ride Park, Ascot \\ SL5 8BP, United Kingdom, Department of Chemistry and Chemical Biology, Harvard \\ University, Cambridge, MA 02138, and Department of Physics, University of Basel, 4056 \\ Basel, Switzerland \\ E-mail: ychu@physics.harvard.edu
}

\begin{abstract}
We report the observation of stable optical transitions in nitrogen-vacancy (NV) centers created by ion implantation. Using a combination of high temperature annealing and subsequent surface treatment, we reproducibly create NV centers with zerophonon lines (ZPL) exhibiting spectral diffusion that is close to the lifetime-limited

${ }^{*}$ To whom correspondence should be addressed

${ }^{\dagger}$ Department of Physics, Harvard University, Cambridge, MA 02138

${ }^{\ddagger}$ School of Engineering and Applied Science, Harvard University, Cambridge, MA 02138

`Institute for Quantum Electronics, ETH-Zurich, CH-8093, Switzerland

${ }^{\S}$ Element Six Ltd, Kings Ride Park, Ascot SL5 8BP, United Kingdom

"Department of Chemistry and Chemical Biology, Harvard University, Cambridge, MA 02138

${ }^{\perp}$ Department of Physics, University of Basel, 4056 Basel, Switzerland

\#Y.C. and N.P.d.L contributed equally to this work.
\end{abstract}


optical linewidth. The residual spectral diffusion is further reduced by using resonant optical pumping to maintain the $\mathrm{NV}^{-}$charge state. This approach allows for placement of NV centers with excellent optical coherence in a well-defined device layer, which is a crucial step in the development of diamond-based devices for quantum optics, nanophotonics, and quantum information science.

Keywords: nitrogen vacancy center, spectral diffusion, diamond, implantation, annealing, surface treatment

Notes: The authors declare no competing finacial interest.

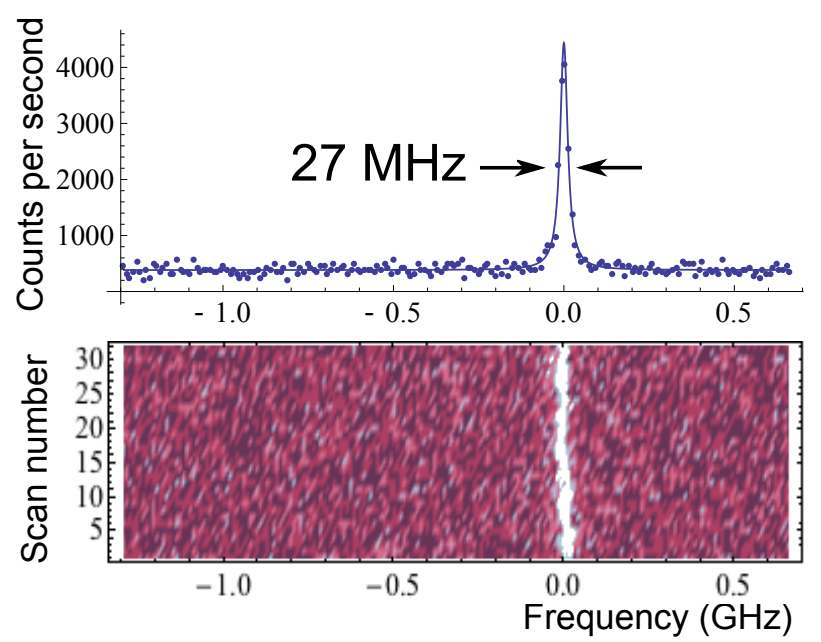


The negatively charged nitrogen vacancy (NV) center in diamond is a solid state system that combines excellent spin coherence with atomic-like optical transitions at cryogenic temperatures. Because of these properties, the NV center has emerged as a promising candidate for the realization of unique applications in the fields of quantum information, quantum optics, and metrology. ${ }^{1-3}$ While many of these applications make use of coherent interactions between light and NV centers, ${ }^{4-6}$ the optical transitions of atom-like solid-state systems are generally imperfect because of the influence of the solid state environment. In particular, the energies of the NV center's optically excited states are highly sensitive to local electric fields. Therefore, fluctuations in the charge environment around the NV center cause fluctuations in the frequency of the optical transition. This phenomenon, which is referred to as spectral diffusion, is a generic effect associated with optical transitions in atom-like solid state systems. ${ }^{7,8}$ Techniques to suppress and eliminate spectral diffusion are currently being actively explored. ${ }^{5,9,10}$

To investigate the optical properties of NV centers, photoluminescence excitation (PLE) studies are typically performed by repeatedly scanning a resonant excitation laser through the NV center's zero-phonon line (ZPL). ${ }^{9-16}$ A $532 \mathrm{~nm}$ laser is applied at the end of each scan to reverse potential photoionization of the NV center caused by the resonant laser. For a single PLE scan, one typically observes a narrow resonance. A nearly lifetime-limited single-scan linewidth of $16 \mathrm{MHz}$ has been reported in a single diamond nanocrystal, ${ }^{11}$ and a lifetime-limited single-scan linewidth of $13 \mathrm{MHz}$ was measured for a native NV center inside a natural diamond sample. ${ }^{12}$ However, these measurements of the single-scan linewidth exclude the effects of spectral diffusion. It was observed that the narrow resonance jumps to a different frequency for each successive PLE scan, indicative of local charge fluctuations caused by the repumping laser. ${ }^{11,13,14}$ Previously, it has been shown that off-resonant excitation can photoionize localized defects inside and on the surface of the diamond crystal, leading to the observed spectral jumps. ${ }^{9,17}$ When the NV center is monitored over long timescales, such behavior results in an overall linewidth that can be much larger than the 
single-scan linewidth. This long-term, extrinsically broadened linewidth, rather than the single-scan linewidth, is the limiting factor for most applications requiring spectral stability. For example, remote entanglement of NV centers relies on interference of photons with well defined matching frequencies. ${ }^{5}$ Efficient photonic devices such as single photon switches and transistors make use of strong NV-photon interactions and require that the emitters remain on resonance with the incident light. ${ }^{18}$ Therefore, a number of different techniques have been explored to reduce the effects of spectral diffusion, including active stabilization and pre-selection of the transition frequency. ${ }^{5,10}$ However, all of these techniques increase the technical complexity and limit the repetition rate of the experiments. The "on demand" creation of emitters with inherently low spectral diffusion under repeated excitation over long timescales is an outstanding challenge in implementing scalable applications using quantum optics with atom-like systems.

Since one-of-a-kind natural diamond samples such as the one investigated in ${ }^{12}$ cannot be used as reproducible starting materials for scalable systems, recent experiments have made use of NV centers incorporated into high quality synthetic diamond during the growth process. ${ }^{5,10,16}$ In addition to imperfections in their optical coherence, NV centers introduced during the diamond growth process are limited in their usefulness due to their low concentration and random locations. For example, in order to incorporate NV centers into nanophotonic or nanomechanical devices, it is necessary to develop a method to create a controllable concentration of emitters in a well defined device layer while maintaining their excellent optical properties.

The key idea of this work is to create NV centers in a surface layer that exhibit coherent optical transitions with nearly lifetime-limited linewidths even under the effects of spectral diffusion. We accomplish this by first introducing NV centers at a well defined and controllable depth using ion implantation. ${ }^{19}$ We then demonstrate how to suppress the fundamental cause of spectral diffusion by creating a diamond environment that is nearly free of defects that contribute to charge fluctuations. This is acheived through a combination of 
annealing and surface treatments. Finally, we further improve the optical properties of the NV centers by combining our approach with a recently developed technique for preventing photoionization of the remaining charge traps. ${ }^{14}$

Fig. 1 summarizes the experimental procedure to create shallow implanted NV centers with narrow optical lines. As our starting material we use single crystal diamond from Element Six grown using microwave assisted chemical vapor deposition (CVD). The single crystal samples were homoepitaxial grown on specially prepared $\langle 100\rangle$ oriented synthetic diamond substrates, taking care to ensure that the surface quality of the substrates was as high as possible to reduce sources of dislocations. The CVD synthesis was performed using conditions as described in. ${ }^{20,21}$ The resultant diamond material is measured using electron paramagnetic resonance $(\mathrm{EPR})$ to have a nitrogen concentration of less than 5 parts per billion. The surface layer of these samples is generally damaged and highly strained because of mechanical polishing. Therefore, we first remove the top several micrometers of the sample using a $30 \mathrm{~min} \mathrm{Ar} / \mathrm{Cl}_{2}$ etch followed by a $20 \mathrm{~min} \mathrm{O}_{2}$ etch in an UNAXIS Shuttleline inductively coupled plasma reactive ion etcher. We then implant ${ }^{15} \mathrm{~N}^{+}$ions at an energy of 85 $\mathrm{keV}$ at a variety of doses from $10^{9} / \mathrm{cm}^{2}$ to $10^{12} / \mathrm{cm}^{2}$. According to simulations done using the Stopping and Range of Ions in Matter (SRIM) software, this should result in a mean nitrogen stopping depth of $\sim 100 \mathrm{~nm}$ with a straggle of $\sim 20 \mathrm{~nm} .{ }^{22}{ }^{15} \mathrm{~N}^{+}$were used instead of ${ }^{14} \mathrm{~N}^{+}$ so that, if needed, we could distinguish NV centers formed from implanted nitrogen versus native nitrogen through electron spin resonance measurements. Subsequently, we anneal the implanted samples under high vacuum $\left(P \lesssim 10^{-6}\right.$ Torr $)$. Our standard annealing recipe consists of a 4 hour $400{ }^{\circ} \mathrm{C}$ step, a 2 hour $800{ }^{\circ} \mathrm{C}$ step, and a 2 hour $1200{ }^{\circ} \mathrm{C}$ step, with a 1 hour ramp up to each temperature. It is important that a good vacuum is maintained throughout the annealing process, since diamond etches and graphitizes under residual oxidizing gasses at such high temperatures. ${ }^{23}$ Following the anneal, we remove graphitic carbon and other surface contaminants by cleaning the diamonds for one hour in a 1:1:1 refluxing mixture of sulfuric, nitric, and perchloric acids. Finally, we perform a $465{ }^{\circ} \mathrm{C}$ annealing in an $\mathrm{O}_{2}$ 
atmosphere in a rapid thermal processor (Modular Process Technology, RTP-600xp) in three 48 minute steps following the recipe used in. ${ }^{24}$ This oxygen annealing is believed to remove $s p^{2}$ hybridized carbon and result in a more perfect oxygen termination of the surface than acid cleaning alone. We have observed that it enhances charge stability in the $\mathrm{NV}^{-}$state and helps to reduce blinking and photobleaching.

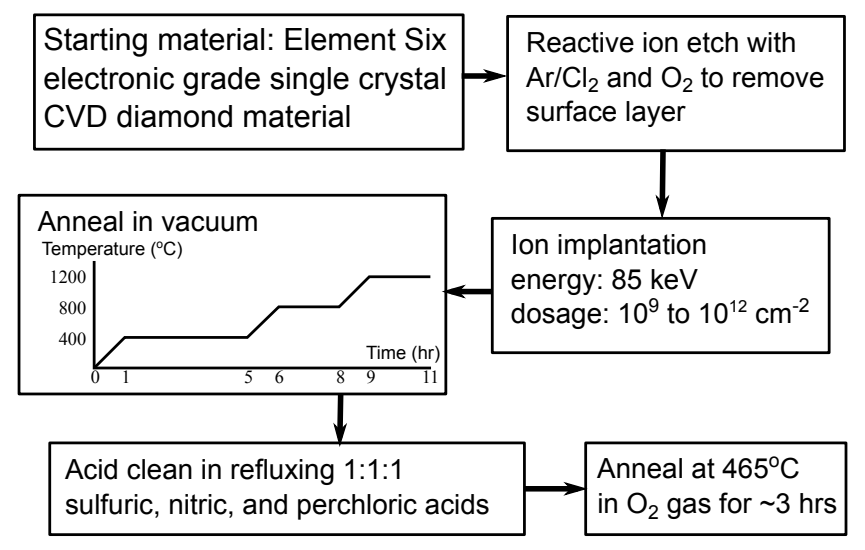

Figure 1: Procedure for creating spectrally stable NV centers through ion implantation, annealing, and surface treatment. The $\mathrm{Ar} / \mathrm{Cl}_{2}\left(\mathrm{O}_{2}\right)$ etch was done using an ICP power of $400 \mathrm{~W}(700 \mathrm{~W})$, a RF power of $250 \mathrm{~W}(100 \mathrm{~W})$, a DC bias of $423 \mathrm{~V}(170 \mathrm{~V})$, a chamber pressure of 8 mTorr (10 mTorr), and a gas flow rate of $25 / 40 \mathrm{sccm}(30 \mathrm{sccm})$. The sample temperature was set to $17^{\circ} \mathrm{C}$.

We investigate the resulting NV centers using a low-temperature confocal microscopy setup, where the diamond samples are cooled to $<10 \mathrm{~K}$ in a helium flow cryostat (Janis ST-500). Fig. 2(a) shows confocal photoluminescence (PL) images taken with $\sim 1 \mathrm{~mW} 532$ $\mathrm{nm}$ laser excitation for four different implantation doses. An implantation dose of $10^{9} / \mathrm{cm}^{2}$ results in a very low density of NV centers, comparable to the native NV concentration in some electronic grade samples. A dose of $10^{12} / \mathrm{cm}^{2}$ results in a high density layer of completely unresolvable NV centers. At an intermediate dose of $10^{10} / \mathrm{cm}^{2}$, however, we obtain resolvable single $\mathrm{NV}$ centers with a density on the order of $1 \mathrm{NV} / \mu \mathrm{m}^{2}$, implying a conversion efficiency of $\sim 1 \%$. Comparing the fluorescence intensity to the single NV count rate, we can roughly estimate that, in the $10^{11} / \mathrm{cm}^{2}$ sample, there are $\sim 10 \mathrm{NV}$ centers per focal spot, while in the $10^{12} / \mathrm{cm}^{2}$ sample, there are $\sim 100 \mathrm{NV}$ centers per focal spot. We note 
that the conversion efficiency is somewhat lower than previously reported values, which could result from differences in starting material, dose calibration, and annealing procedures. ${ }^{19}$

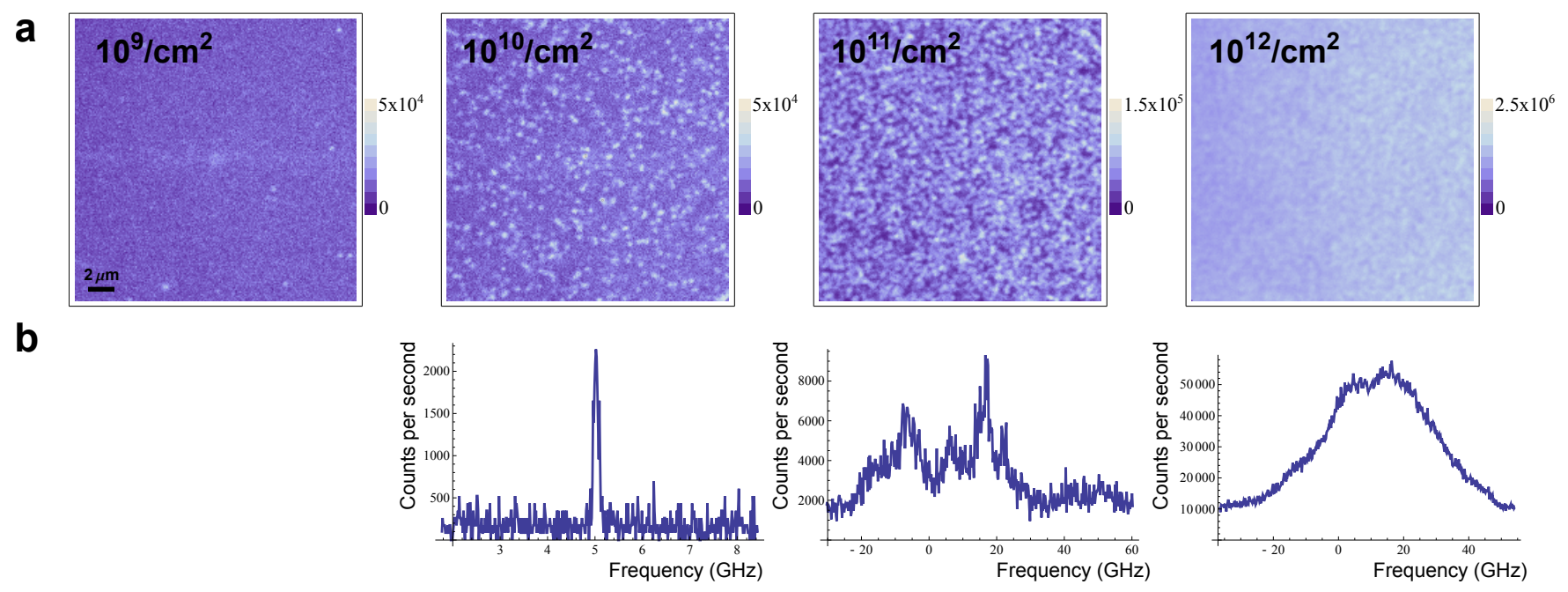

Figure 2: (a) Confocal PL images of the implanted layer of NV centers for four different nitrogen ion doses. (b) Corresponding representative PLE spectra for the three highest doses. Each spectrum is the average of $~ 10$ PLE scans

In Fig. 2(b), we present corresponding ZPL spectra of NV centers for the three highest implantation doses. The spectra were taken by scanning an external cavity diode laser around $637.2 \mathrm{~nm}$ while collecting fluorescence in the phonon-sideband (PSB). $532 \mathrm{~nm}$ excitation was performed at each point of the scan to alleviate the effects of photoionization during resonant excitation. Without such repumping to maintain the charge state, we find that most NV centers remain in the $\mathrm{NV}^{-}$charge state for a few PLE scans and demonstrate almost no spectral diffusion. Subsequently, the NV center ionizes and remains dark. We emphasize here that Fig. 2(b) and Fig. 3(b-d) present spectra that are averages of many successive laser scans taken over the course of minutes. Therefore, they are indicative of the spectral stability of NV centers over both short and long time scales and include all effects of spectral diffusion caused by the repumping laser. Beyond these timescales, longer-term drifts of the NV frequency can be easily compensated by electric fields or tuning the excitation frequency with minimal cost to the experimental repetition rate. At a dose of $10^{10} / \mathrm{cm}^{2}$, most single NV centers exhibit narrow PLE lines. As the dose increases to $10^{11} / \mathrm{cm}^{2}$, the spectra show 
multiple features with several broader peaks. Finally, at $10^{12} / \mathrm{cm}^{2}$, the spectrum is a mostly featureless peak several tens of $\mathrm{GHz}$ wide. We note here that the spectra for the two highest doses exhibit the effects of both increased single NV center linewidths and the presence of several NV centers in the focal spot. If the linewidths were comparable to the $10^{10} / \mathrm{cm}^{2}$ sample, we should be able to observe a collection of narrow resonances spread over the scan range. However, this is not the case, which is an indication that increased nitrogen concentration in the environment of the NV center leads to additional spectral diffusion. Indeed, the substitutional nitrogen defect has an ionization energy threshold of $2.2 \mathrm{eV}$ and therefore can be photoionized with $532 \mathrm{~nm}$ light, which has an energy of $2.33 \mathrm{eV} /$ photon. $^{25}$

From this point on, we focus on samples implanted with a dose of $10^{10} / \mathrm{cm}^{2}$. Fig. 3(a) shows a histogram of linewidths for $50 \mathrm{NV}$ centers in four such samples. While there is a range of linewidths, a majority of NV centers exhibit linewidths less than $500 \mathrm{MHz}$, which is comparable to naturally occurring NV centers deep inside electronic grade CVD diamond. ${ }^{5}$ This is an indication that we have eliminated most impurities with fluctuating charge states in the vicinity of the NV centers, which may include both impurities on the nearby surface and defects inside the diamond introduced by the implantation process. In Fig. 3(b), we present an example spectrum of the narrowest linewidth we have observed, along with the results of twenty-one successive PLE scans to demonstrate the long-term stability of the transition line. Our measured linewidth of $37 \pm 5 \mathrm{MHz}$ under conventional $532 \mathrm{~nm}$ repumping is, to the best of our knowledge, a record for the extrinsically broadened linewidth of NV centers.

To further reduce residual spectral diffusion of the NV centers, we incorporate a technique recently demonstrated in. ${ }^{14}$ Instead of using $532 \mathrm{~nm}$ light to repump the $\mathrm{NV}^{0}$ state into $\mathrm{NV}^{-}$, we use a home-built tunable frequency doubled external cavity diode laser operating at 575 nm to resonantly excite the $\mathrm{NV}^{0} \mathrm{ZPL}$, which has been shown to efficiently convert the NV center back to the negatively charged state even at very low powers. The $575 \mathrm{~nm}$ laser was turned on and scanned across the $\mathrm{NV}^{0} \mathrm{ZPL}$ between each frequency scan of the $637 \mathrm{~nm}$ spectroscopy laser. As was also demonstrated in, ${ }^{14}$ this method results in a drastic reduction 
of the spectral diffusion because of the lower incident intensity and energy per photon of the $575 \mathrm{~nm}$ excitation $(2.16 \mathrm{eV} /$ photon) compared to the conventional $532 \mathrm{~nm}$ excitation. For example, the NV center shown in Fig. 3(c) has a NV- ZPL linewidth of $161 \mathrm{MHz}$ using 532 $\mathrm{nm}$ excitation. The linewidth was reduced to $27 \mathrm{MHz}$ using the $575 \mathrm{~nm}$ repumping technique (Fig. 3(d)).
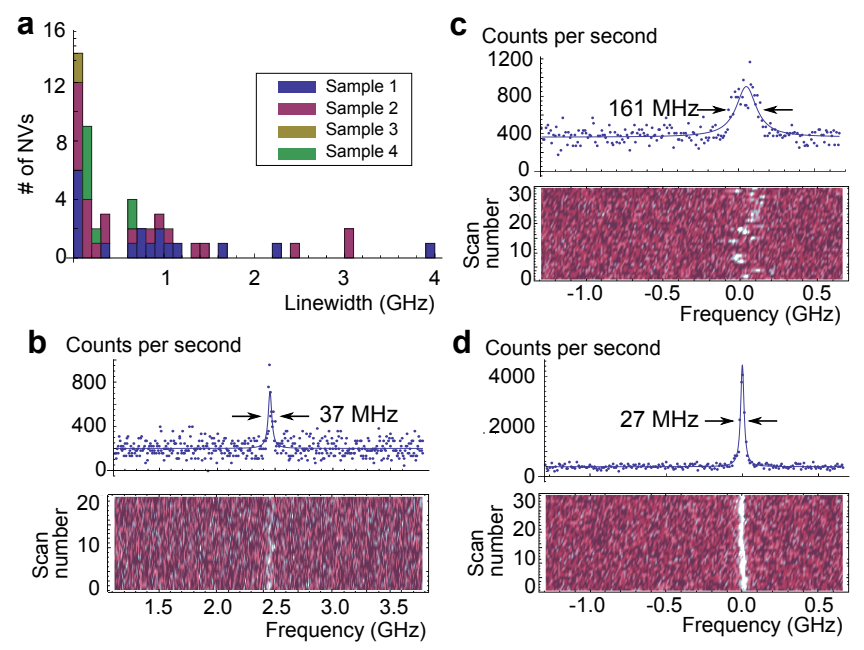

Figure 3: (a) Histogram of linewidths for NV centers in four samples implanted with a dose of $10^{10} / \mathrm{cm}^{2}$ using $532 \mathrm{~nm}$ excitation to repump the NV into the negative charge state. (b) Spectrum showing the narrowest linewidth from (a) (top) obtained by averaging twenty-one successive PLE scans (bottom). Each scan takes $\sim 2$ seconds. (c) Spectrum of another NV taken using $532 \mathrm{~nm}$ repump (top). Corresponding single PLE scans illustrating spectral jumps due to repumping (bottom). (d) Spectrum of the same NV as in (c), taken using 575 $\mathrm{nm}$ resonant excitation of the $\mathrm{NV}^{0}$ state to repump back into $\mathrm{NV}^{-}$.

We now discuss our sample preparation process in more detail and propose a mechanism for the creation of optically stable NV centers through high temperature annealing. In general, annealing is required after ion implantation to mobilize vacancies such that they combine with the nitrogen atoms to form NV centers. In addition, the annealing process causes other defects to become mobile at particular temperatures and potentially migrate to the surface, effectively repairing any damage to the crystal caused by implantation. Earlier works have employed a range of different annealing temperatures, typically in the range between $400{ }^{\circ} \mathrm{C}$ and $1000{ }^{\circ} \mathrm{C}$. Several temperature steps in this range were shown to enable specific mechanisms important to the efficient formation of stable NV centers. In particular, 
at $400{ }^{\circ} \mathrm{C}$, earlier work has indicated that vacancies and interstitial nitrogen atoms introduced by implantation migrate and annihilate with each other. ${ }^{26}$ At $800{ }^{\circ} \mathrm{C}$, vacancies become mobile and combine with nitrogen atoms on lattice sites to form NV centers, which then remain stable. ${ }^{27}$ At temperatures much less than $800{ }^{\circ} \mathrm{C}$, this process is too slow to allow $\mathrm{NV}$ formation in a reasonable time period. At temperatures much higher than $800{ }^{\circ} \mathrm{C}$, the vacancies are highly mobile, but there is indication that the rate of $\mathrm{NV}$ formation is suppressed. ${ }^{28}$ This could be because of the higher thermal energy in the diamond lattice, which causes the local strain field around the isolated nitrogen defects to becomes less efficient at trapping migrating vacancies.

At even higher temperatures, additional defects such as divacancies, $\mathrm{sp}^{2}$ type defects, and hydrogen become mobile and anneal out. ${ }^{27,29,30}$ Recent work has demonstrated that annealing at a temperature of $1200{ }^{\circ} \mathrm{C}$ significantly improved the coherence properties of implanted NV centers. ${ }^{31}$ This indicates that some of the remaining defects after $800{ }^{\circ} \mathrm{C}$ annealing are paramagnetic. The electronic and optical properties of these defects, such as the level structure and ionization energies, have generally not been well studied. However, we have briefly investigated the optical properties of samples annealed without the $1200{ }^{\circ} \mathrm{C}$ step and found that, while a few NV centers had linewidths greater than several hundred $\mathrm{MHz}$, most had PLE lines that were too broad to be discernible. These results provide evidence that annealing at high temperatures eliminates localized defects that can affect the optical properties of NV centers. The exact structure and properties of these defect states remain to be determined through, for example, EPR measurements, optical spectroscopy, and ab initio calculations of energy level structures.

Our work shows that it is possible to create implanted NV centers with excellent optical coherence properties, opening up a large range of possibilities for future studies and applications. In addition to a better understanding of the material and defect properties that contribute to spectral diffusion, there are many other aspects and parameters of our technique that remain to be explored. For example, as mentioned above, we believe that 
high temperature annealing is crucial for the creation of high quality NV centers. However, it may be possible to modify the exact temperature and time of each annealing step to achieve similar or even better results. Furthermore, while we have not extensively studied the dependence of optical coherence on implantation depth, there is evidence that reasonably stable transitions are possible with much shallower NV centers. We have observed linewidths of a few hundred $\mathrm{MHz}$ from several NV centers in a diamond sample created by implantation with a nitrogen dose of $10^{11} / \mathrm{cm}^{2}$ at an energy of $6 \mathrm{keV}$, giving a nominal depth of $10 \mathrm{~nm}$. This also indicates that our sample processing method does not remove more than a few nanometers from the diamond surface. Further systematic studies on the effect of implantation energy, dose, and annealing recipes are needed to explore the possibility of creating optically coherent NV centers in high densities or very close to the diamond surface for applications such as coupling of multiple NV centers and electric field sensing.

Another important application that has recently become the focus of much effort is the implementation of nanoscale photonic structures to tailor the interaction of light and NV centers. ${ }^{32}$ Thus far, all previous demonstrations of diamond-based photonic structures have used diamond materials with a high density of native NV centers, whose linewidths are generally many $\mathrm{GHz}$ or more. ${ }^{32,33}$ To realize quantum nanophotonic devices in deterministic, scalable fashion, it is important to begin with a bulk material that contains very high quality emitters in a well-defined device layer. For this reason, the ability to controllably create NV centers with inherently good optical coherence, which we have demonstrated in this work, is a crucial step for the practical development of many diamond-based quantum nanophotonic devices and scalable quantum technologies.

\section{Acknowledgement}

We thank F. Jelezko for many fruitful discussions and for sharing the ideas on $\mathrm{NV}^{0}$ repumping technique with us. Etching and post-processing of diamond samples were performed in the Center for Nanoscale Systems at Harvard. This work was supported by the NSF, CUA, 
and the DARPA QuASAR and SPARQC programs. Y.C. and R.E. acknowledge financial support from the NSF GRFP. N.P.d.L. acknowledges financial support from the Element Six postdoctoral fellowship. B.H. and Y.C. acknowledges financial support from HQOC.

\section{References}

(1) Bernien, H.; Hensen, B.; Pfaff, W.; Koolstra, G.; Blok, M. S.; Robledo, L.; Taminiau, T. H.; Markham, M.; Twitchen, D. J.; Childress, L.; Hanson, R. Nature 2013, 497, 86-90.

(2) Balasubramanian, G.; Chan, I. Y.; Kolesov, R.; Al-Hmoud, M.; Tisler, J.; Shin, C.; Kim, C.; Wjcik, A.; Hemmer, P. R.; Krueger, A.; Hanke, T.; Leitenstorfer, A.; Bratschitsch, R.; Jelezko, F.; Wrachtrup, J. Nature 2008, 455, 648-651.

(3) Maurer, P. C.; Kucsko, G.; Latta, C.; Jiang, L.; Yao, N. Y.; Bennett, S. D.; Pastawski, F.; Hunger, D.; Chisholm, N.; Markham, M.; Twitchen, D. J.; Cirac, J. I.; Lukin, M. D. Science 2012, 336, 1283-1286.

(4) Togan, E.; Chu, Y.; Trifonov, A. S.; Jiang, L.; Maze, J.; Childress, L.; Dutt, M. V. G.; Sorensen, A. S.; Hemmer, P. R.; Zibrov, A. S.; Lukin, M. D. Nature 2010, 466, 730-734.

(5) Bernien, H.; Childress, L.; Robledo, L.; Markham, M.; Twitchen, D.; Hanson, R. Phys. Rev. Lett. 2012, 108, 043604.

(6) Sipahigil, A.; Goldman, M. L.; Togan, E.; Chu, Y.; Markham, M.; Twitchen, D. J.; Zibrov, A. S.; Kubanek, A.; Lukin, M. D. Phys. Rev. Lett. 2012, 108, 143601.

(7) Robinson, H. D.; Goldberg, B. B. Phys. Rev. B 2000, 61, R5086-R5089.

(8) Ambrose, W. P.; Moerner, W. E. Nature 1991, 349, $225-227$.

(9) Bassett, L. C.; Heremans, F. J.; Yale, C. G.; Buckley, B. B.; Awschalom, D. D. Phys. Rev. Lett. 2011, 107, 266403. 
(10) Acosta, V. M.; Santori, C.; Faraon, A.; Huang, Z.; Fu, K.-M. C.; Stacey, A.; Simpson, D. A.; Ganesan, K.; Tomljenovic-Hanic, S.; Greentree, A. D.; Prawer, S.; Beausoleil, R. G. Phys. Rev. Lett. 2012, 108, 206401.

(11) Shen, Y.; Sweeney, T. M.; Wang, H. Phys. Rev. B 2008, 77, 033201.

(12) Tamarat, P.; Gaebel, T.; Rabeau, J. R.; Khan, M.; Greentree, A. D.; Wilson, H.; Hollenberg, L. C. L.; Prawer, S.; Hemmer, P.; Jelezko, F.; Wrachtrup, J. Phys. Rev. Lett. 2006, 97, 083002.

(13) Fu, K.-M. C.; Santori, C.; Barclay, P. E.; Rogers, L. J.; Manson, N. B.; Beausoleil, R. G. Phys. Rev. Lett. 2009, 103, 256404.

(14) Siyushev, P.; Pinto, H.; Vörös, M.; Gali, A.; Jelezko, F.; Wrachtrup, J. Phys. Rev. Lett. 2013, 110, 167402.

(15) Orwa, J. O.; Santori, C.; Fu, K. M. C.; Gibson, B.; Simpson, D.; Aharonovich, I.; Stacey, A.; Cimmino, A.; Balog, P.; Markham, M.; Twitchen, D.; Greentree, A. D.; Beausoleil, R. G.; Prawer, S. J. Appl. Phys. 2011, 109, 083530.

(16) Stacey, A.; Simpson, D. A.; Karle, T. J.; Gibson, B. C.; Acosta, V. M.; Huang, Z.; Fu, K. M. C.; Santori, C.; Beausoleil, R. G.; McGuinness, L. P.; Ganesan, K.; TomljenovicHanic, S.; Greentree, A. D.; Prawer, S. Adv. Mater. 2012, 24, 3333-3338.

(17) Aslam, N.; Waldherr, G.; Neumann, P.; Jelezko, F.; Wrachtrup, J. New J. Phys. 2013, 15,013064 .

(18) Chang, D. E.; Sorensen, A. S.; Demler, E. A.; Lukin, M. D. Nat. Phys. 2007, 3, 807-812.

(19) Pezzagna, S.; Naydenov, B.; Jelezko, F.; Wrachtrup, J.; Meijer, J. New J. Phys. 2010, 12, 065017.

(20) Isberg, J.; Hammersberg, J.; Johansson, E.; Wikstršm, T.; Twitchen, D. J.; Whitehead, A. J.; Coe, S. E.; Scarsbrook, G. A. Science 2002, 297, 1670-1672. 
(21) Markham, M.; Dodson, J.; Scarsbrook, G.; Twitchen, D.; Balasubramanian, G.; Jelezko, F.; Wrachtrup, J. Diamond Relat. Mater. 2011, 20, 134 - 139.

(22) Ziegler, J. F. Particle Interactions With Matter. 2013; http://www.srim.org.

(23) Seal, M. Phys. Status Solidi B 1963, 3, 658-664.

(24) Fu, K.-M. C.; Santori, C.; Barclay, P. E.; Beausoleil, R. G. Appl. Phys. Lett. 2010, 96, 121907.

(25) Rosa, J.; Vanecek, M.; Nesladek, M.; Stals, L. Diamond Relat. Mater. 1999, 8, 721-724.

(26) Twitchen, D.; Hunt, D.; Wade, C.; Newton, M.; Baker, J.; Anthony, T.; Banholzer, W. Phys. B 1999, $273-274,644-646$.

(27) Twitchen, D. J.; Newton, M. E.; Baker, J. M.; Anthony, T. R.; Banholzer, W. F. Phys. Rev. B 1999, 59, 12900-12910.

(28) Twitchen, D. J.; Markham, M. 2013; Personal communication.

(29) Lea-wilsonf, M. A.; Lomer, J. N.; Van Wyk, J. A. Philos. Mag. B 1995, 72, 81-89.

(30) Talbot-Ponsonby, D. F.; Newton, M. E.; Baker, J. M.; Scarsbrook, G. A.; Sussmann, R. S.; Whitehead, A. J. Phys. Rev. B 1998, 57, 2302-2309.

(31) Naydenov, B.; Reinhard, F.; Laemmle, A.; Richter, V.; Kalish, R.; D’HaenensJohansson, U. F. S.; Newton, M.; Jelezko, F.; Wrachtrup, J. Appl. Phys. Lett. 2010, 97, 242511.

(32) Faraon, A.; Santori, C.; Huang, Z.; Acosta, V. M.; Beausoleil, R. G. Phys. Rev. Lett. 2012, 109, 033604 .

(33) Babinec, T. M.; Hausmann, B. J. M.; Khan, M.; Zhang, Y.; Maze, J. R.; Hemmer, P. R.; Loncar, M. Nat. Nanotechnol. 2010, 5, 195-199. 